\title{
Parallel Breadth-First Search on Distributed Memory Systems
}

\author{
Aydın Buluç Kamesh Madduri \\ Computational Research Division \\ Lawrence Berkeley National Laboratory \\ Berkeley, CA \\ \{ABuluc, KMadduri\}@lbl.gov
}

\begin{abstract}
Data-intensive, graph-based computations are pervasive in several scientific applications, and are known to to be quite challenging to implement on distributed memory systems. In this work, we explore the design space of parallel algorithms for Breadth-First Search (BFS), a key subroutine in several graph algorithms. We present two highly-tuned parallel approaches for BFS on large parallel systems: a levelsynchronous strategy that relies on a simple vertex-based partitioning of the graph, and a two-dimensional sparse matrix partitioning-based approach that mitigates parallel communication overhead. For both approaches, we also present hybrid versions with intra-node multithreading. Our novel hybrid two-dimensional algorithm reduces communication times by up to a factor of 3.5 , relative to a common vertex based approach. Our experimental study identifies execution regimes in which these approaches will be competitive, and we demonstrate extremely high performance on leading distributed-memory parallel systems. For instance, for a 40,000-core parallel execution on Hopper, an AMD MagnyCours based system, we achieve a BFS performance rate of 17.8 billion edge visits per second on an undirected graph of 4.3 billion vertices and 68.7 billion edges with skewed degree distribution.
\end{abstract}

\section{INTRODUCTION}

The use of graph abstractions to analyze and understand social interaction data, complex engineered systems such as the power grid and the Internet, communication data such as email and phone networks, biological systems, and in general, various forms of relational data, has been gaining ever-increasing importance. Common graph-theoretic problems arising in these application areas include identifying and ranking important entities, detecting anomalous patterns or sudden changes in networks, finding tightly interconnected clusters of entities, and so on. The solutions to these problems typically involve classical algorithms for problems such as finding spanning trees, shortest paths, biconnected components, matchings, flow-based computa-

Permission to make digital or hard copies of all or part of this work for personal or classroom use is granted without fee provided that copies are not made or distributed for profit or commercial advantage and that copies bear this notice and the full citation on the first page. To copy otherwise, to republish, to post on servers or to redistribute to lists, requires prior specific permission and/or a fee.

SC11, November 12-18, 2011, Seattle, Washington, USA

Copyright 2011 ACM 978-1-4503-0771-0/11/11 ...\$10.00. tions, in these graphs. To cater to the graph-theoretic analyses demands of emerging "big data" applications, it is essential that we speed up the underlying graph problems on current parallel systems.

We study the problem of traversing large graphs in this paper. A traversal refers to a systematic method of exploring all the vertices and edges in a graph. The ordering of vertices using a "breadth-first" search (BFS) is of particular interest in many graph problems. Theoretical analysis in the random access machine (RAM) model of computation indicates that the computational work performed by an efficient BFS algorithm would scale linearly with the number of vertices and edges, and there are several well-known serial and parallel BFS algorithms (discussed in Section 2). However, efficient RAM algorithms do not easily translate into "good performance" on current computing platforms. This mismatch arises due to the fact that current architectures lean towards efficient execution of regular computations with low memory footprints, and heavily penalize memory-intensive codes with irregular memory accesses. Graph traversal problems such as BFS are by definition predominantly memory access-bound, and these accesses are further dependent on the structure of the input graph, thereby making the algorithms "irregular".

The recently-created Graph 500 List [20] is an attempt to rank supercomputers based on their performance on dataintensive applications, and BFS is chosen as the first representative benchmark. Distributed memory architectures dominate the supercomputer market and computational scientists have an excellent understanding on mapping numerical scientific applications to these systems. Measuring the sustained floating point execution rate (Flop/s) and comparison with the theoretical system peak is a popular technique. In contrast, little is known about the design and parallel performance of data-intensive graph algorithms, and the programmability trade-offs involved. Thus, the current Graph 500 list does not yet accurately portray the data-crunching capabilities of parallel systems, and is just a reflection of the quality of various benchmark BFS implementations.

BFS on distributed-memory systems involves explicit communication between processors, and the distribution (or partitioning) of the graph among processors also impacts performance. We utilize a testbed of large-scale graphs with billions of vertices and edges to empirically evaluate the performance of our BFS algorithms. These graphs are all sparse, i.e., the number of edges $m$ is just a constant factor times the number of vertices $n$. Further, the average path length in these graphs is a small constant value compared 
to the number of vertices, or is at most bounded by $\log n$.

\section{Our Contributions}

We present two complementary approaches to distributedmemory BFS on graphs with skewed degree distribution. The first approach is a more traditional scheme using onedimensional distributed adjacency arrays for representing the graph. The second method utilizes a sparse matrix representation of the graph and a two-dimensional partitioning among processors. The following are our major contributions:

- Our two-dimensional partitioning-based approach, coupled with intranode multithreading, reduces the communication overhead at high process concurrencies by a factor of 3.5 .

- Both our approaches include extensive intra-node multicore tuning and performance optimization. The singlenode performance of our graph-based approach is comparable to, or exceeds, recent single-node shared memory results, on a variety of real-world and synthetic networks. The hybrid schemes (distributed memory graph partitioning and shared memory traversal parallelism) enable BFS scalability up to 40,000 cores.

- To accurately quantify the memory access costs in BFS, we present a simple memory-reference centric performance model. This model succinctly captures the differences between our two BFS strategies, and also provides insight into architectural trends that enable high-performance graph algorithms.

\section{BREADTH-FIRST SEARCH OVERVIEW}

\subsection{Preliminaries}

Given a distinguished "source vertex" $s$, Breadth-First Search (BFS) systematically explores the graph $G$ to discover every vertex that is reachable from $s$. Let $V$ and $E$ refer to the vertex and edge sets of $G$, whose cardinalities are $n=|V|$ and $m=|E|$. We assume that the graph is unweighted; equivalently, each edge $e \in E$ is assigned a weight of unity. A path from vertex $s$ to $t$ is defined as a sequence of edges $\left\langle u_{i}, u_{i+1}\right\rangle$ (edge directivity assumed to be $u_{i} \rightarrow u_{i+1}$ in case of directed graphs), $0 \leq i<l$, where $u_{0}=s$ and $u_{l}=t$. The length of a path is the sum of the weights of edges. We use $d(s, t)$ to denote the distance between vertices $s$ and $t$, or the length of the shortest path connecting $s$ and $t$. BFS implies that all vertices at a distance $k$ (or "level" $k$ ) from vertex $s$ should be first "visited" before vertices at distance $k+1$. The distance from $s$ to each reachable vertex is typically the final output. In applications based on a breadth-first graph traversal, one might optionally perform auxiliary computations when visiting a vertex for the first time. Additionally, a "breadth-first spanning tree" rooted at $s$ containing all the reachable vertices can also be maintained.

Algorithm 1 gives a serial algorithm for BFS. The required breadth-first ordering of vertices is accomplished in this case by using two stacks - FS and $N S$ - for storing vertices at the current level (or "frontier") and the newly-visited set of vertices (one hop away from the current level) respectively. The number of iterations of the outer while loop (lines 511) is bounded by the length of the longest shortest path from $s$ to any reachable vertex $t$. Note that this algorithm

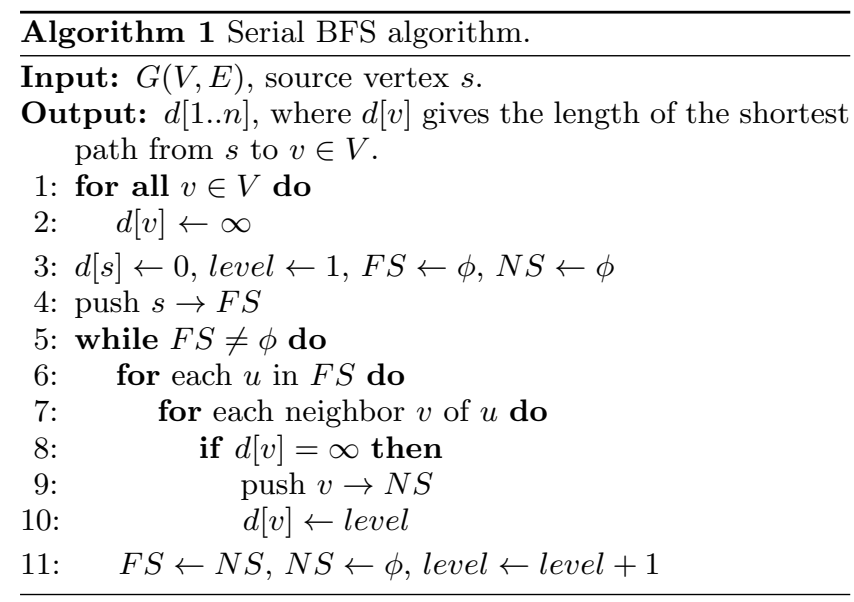

is slightly different from the widely-used queue-based serial algorithm [14]. We can relax the FIFO ordering mandated by a queue at the cost of additional space utilization, but the work complexity in the RAM model is still $O(m+n)$.

\subsection{Parallel BFS: Prior Work}

Parallel algorithms for BFS date back to nearly three decades [31,32]. The classical parallel random access machine (PRAM) approach to BFS is a straightforward extension of the serial algorithm presented in Algorithm 1. The graph traversal loops (lines 6 and 7 ) are executed in parallel by multiple processing elements, and the distance update and stack push steps (lines 8-10) are atomic. There is a barrier synchronization step once for each level, and thus the execution time in the PRAM model is $O(D)$, where the $D$ is the diameter of the graph. Since the PRAM model does not weigh in synchronization costs, the asymptotic complexity of work performed is identical to the serial algorithm.

The majority of the novel parallel implementations developed for BFS follow the general structure of this "levelsynchronous" algorithm, but adapt the algorithm to better fit the underlying parallel architecture. In addition to keeping the parallel work complexity close to $O(m+n)$, the three key optimization directions pursued are

- ensuring that parallelization of the edge visit steps (lines 6, 7 in Algorithm 1) is load-balanced,

- mitigating synchronization costs due to atomic updates and the barrier synchronization at the end of each level, and

- improving locality of memory references by modifying the graph layout and/or BFS data structures.

We discuss recent work on parallel BFS in this section, and categorize them based on the parallel system they were designed for.

Multithreaded systems: Bader and Madduri [4] present a fine-grained parallelization of the above level-synchronous algorithm for the Cray MTA-2, a massively multithreaded shared memory parallel system. Their approach utilizes the support for fine-grained, low-overhead synchronization provided on the MTA-2, and ensures that the graph traversal is load-balanced to run on thousands of hardware threads. The MTA-2 system is unique in that it relies completely on hardware multithreading to hide memory latency, as there are no data caches in this system. This feature also eliminates the necessity of tedious locality-improvement optimizations to the BFS algorithm, and Bader and Madduri's 
implementation achieves a very high system utilization on a 40-processor MTA-2 system. Mizell and Maschhoff [29] discuss an improvement to the Bader-Madduri MTA-2 approach, and present performance results for parallel BFS on a 128-processor Cray XMT system, a successor to the Cray MTA-2.

The current generation of GPGPUs are similar to the Cray XMT systems in their reliance on large-scale multithreading to hide memory latency. In addition, one needs to ensure that the threads perform regular and contiguous memory accesses to achieve high system utilization. This makes optimizing BFS for these architectures quite challenging, as there is no work-efficient way to ensure coalesced accesses to the $d$ array in the level synchronous algorithm. Harish and Narayanan [22] discuss implementations of BFS and other graph algorithms on NVIDIA GPUs. Due to the comparably higher memory bandwidth offered by the GDDR memory, they show that the GPU implementations outperform BFS approaches on the CPU for various low-diameter graph families with tens of millions of vertices and edges. Luo et al. [27] present an improvement to this work with a new hierarchical data structure to store and access the frontier vertices, and demonstrate that their algorithm is up to $10 \times$ faster than the Harish-Narayanan algorithm on recent NVIDIA GPUs and low-diameter sparse graphs. You et al. [39] study BFSlike traversal optimization on GPUs and multicore CPUs in the context of implementing an inference engine for a speech recognition application.

Multicore systems: There has been a spurt in recent work on BFS approaches for multicore CPU systems. Current x 86 multicore architectures, with 8 to 32 -way core-level parallelism and 2-4 way simultaneous multithreading, are much more amenable to coarse-grained load balancing in comparison to the multithreaded architectures. Possible $p$-way partitioning of vertices and/or replication of highcontention data structures alleviates some of the synchronization overhead. However, due to the memory-intensive nature of BFS, performance is still quite dependent on the graph size, as well as the sizes and memory bandwidths of the various levels of the cache hierarchy. Recent work on parallelization of the queue-based algorithm by Agarwal et al. [1] notes a problem with scaling of atomic intrinsics on multi-socket Intel Nehalem systems. To mitigate this, they suggest a partitioning of vertices and corresponding edges among multiple sockets, and a combination of the fine-grained approach and the accumulation-based approach in edge traversal. In specific, the distance values (or the "visited" statuses of vertices in their work) of local vertices are updated atomically, while non-local vertices are held back to avoid coherence traffic due to cache line invalidations. They achieve very good scaling going from one to four sockets with this optimization, at the expense of introducing an additional barrier synchronization for each BFS level. Xia and Prasanna [37] also explore synchronizationreducing optimizations for BFS on Intel Nehalem multicore systems. Their new contribution is a low-overhead "adaptive barrier" at the end of each frontier expansion that adjusts the number of threads participating in traversal based on an estimate of work to be performed. They show significant performance improvements over naïve parallel BFS implementations on dual-socket Nehalem systems. Leiserson and Schardl [25] explore a different optimization: they replace the shared queue with a new "bag" data structure which is more amenable for code parallelization with the Cilk++ run-time model. These three approaches use seemingly independent optimizations and different graph families to evaluate performance on, which makes it difficult to do a head-to-head comparison. Since our target architecture in this study are clusters of multicore nodes, we share some similarities to these approaches.

Distributed memory systems: The general structure of the level-synchronous approach holds in case of distributed memory implementations as well, but fine-grained "visited" checks are replaced by edge aggregation-based strategies. With a distributed graph and a distributed $d$ array, a processor cannot tell whether a non-local vertex has been previously visited or not. So the common approach taken is to just accumulate all edges corresponding to non-local vertices, and send them to the owner processor at the end of a local traversal. There is thus an additional all-to-all communication step at the end of each frontier expansion. Interprocessor communication is considered a significant performance bottleneck in prior work on distributed graph algorithms $[11,26]$. The relative costs of inter-processor communication and local computation depends on the quality of the graph partitioning and the topological characteristics of the interconnection network.

The BFS implementation of Scarpazza et al. [33] for the Cell/B.E. processor, while being a multicore implementation, shares a lot of similarities with the general "explicit partitioning and edge aggregation" BFS strategy for distributed memory system. The implementation by Yoo et al. [38] for the BlueGene/L system is a notable distributed memory parallelization. The authors observe that a twodimensional graph partitioning scheme would limit key collective communication phases of the algorithms to at most $\sqrt{p}$ processors, thus avoiding the expensive all-to-all communication steps. This enables them to scale BFS to process concurrencies as high as 32,000 processors. However, this implementation assumes that the graph families under exploration would have a regular degree distribution, and computes bounds for inter-process communication message buffers based on this assumption. Such large-scale scalability with or without $2 \mathrm{D}$ graph decomposition may not be realizable for graphs with skewed degree distributions. Furthermore, the computation time increases dramatically (up to 10-fold) with increasing processor counts, under a weak scaling regime. This implies that the sequential kernels and data structures used in this study were not work-efficient. As opposed to Yoo et al.'s work, we give details of the data structures and algorithms that are local to each processor in Section 4. Cong et al. [12] study the design and implementation of several graph algorithms using the partitioned global address space (PGAS) programming model. Recently, Edmonds et al. [16] gave the first hybrid-parallel 1D BFS implementation that uses active messages.

Software systems for large-scale distributed graph algorithm design include the Parallel Boost graph library (PBGL) [21] and the Pregel [28] framework. Both these systems adopt a straightforward level-synchronous approach for BFS and related problems. Prior distributed graph algorithms are predominantly designed for "shared-nothing" settings. However, current systems offer a significant amount of parallelism within a single processing node, with per-node memory capacities increasing as well. Our paper focuses on graph traversal algorithm design in such a scenario. We present 
these new parallel strategies and quantify the performance benefits achieved in Section 3.

External memory algorithms: Random accesses to disk are extremely expensive, and so locality-improvement optimizations are the key focus of external memory graph algorithms. External memory graph algorithms build on known I/O-optimal strategies for sorting and scanning. Ajwani and Meyer [2,3] discuss the state-of-the-art algorithms for BFS and related graph traversal problems, and present performance results on large-scale graphs from several families. Recent work by Pierce et al. [30] investigates implementations of semi-external BFS, shortest paths, and connected components.

Other Parallel BFS Algorithms: There are several alternate parallel algorithms to the level-synchronous approach, but we are unaware of any recent, optimized implementations of these algorithms. The fastest-known algorithm (in the PRAM complexity model) for BFS repeatedly squares the adjacency matrix of the graph, where the element-wise operations are in the min-plus semiring (see [17] for a detailed discussion). This computes the BFS ordering of the vertices in $O(\log n)$ time in the EREW-PRAM model, but requires $O\left(n^{3}\right)$ processors for achieving these bounds. This is perhaps too work-inefficient for traversing large-scale graphs. The level synchronous approach is also clearly inefficient for high-diameter graphs. A PRAM algorithm designed by Ullman and Yannakakis [35], based on path-limited searches, is a possible alternative on sharedmemory systems. However, it is far more complicated than the simple level-synchronous approach, and has not been empirically evaluated. The graph partitioning-based strategies adopted by Ajwani and Meyer [3] in their external memory traversal of high-diameter graphs may possibly lend themselves to efficient in-memory implementations as well.

Other Related Work: Graph partitioning is intrinsic to distributed memory graph algorithm design, as it helps bound inter-processor communication traffic. One can further relabel vertices based on partitioning or other heuristics $[13,15]$, and this has the effect of improving memory reference locality and thus improve parallel scaling.

A sparse graph can analogously be viewed as a sparse matrix, and optimization strategies for linear algebra computations similar to BFS, such as sparse matrix-vector multiplication [36], may be translated to the realm of graph algorithms to improve BFS performance as well. Recent research shows prospects of viewing graph algorithms as sparse matrix operations $[8,19]$. Our work contributes to that area by exploring the use of sparse-matrix sparse-vector multiplication for BFS for the first time.

The formulation of BFS that is common in combinatorial optimization and artificial intelligence search applications [5, $24]$ is different from the focus of this paper.

\section{BREADTH-FIRST SEARCH ON DISTRIBUTED MEMORY SYSTEMS}

In this section, we briefly describe the high-level parallelization strategy employed in our two distributed BFS schemes with accompanying pseudo-code. Section 4 provides more details about the parallel implementation of these algorithms. The algorithms are seemingly straightforward to implement, but eliciting high performance on current systems requires careful data structure choices and low-level performance tun- ing. Section 5.1 provides a rigorous analysis of both parallel implementations.

\subsection{BFS with 1D Partitioning}

A natural way of distributing the vertices and edges of a graph on a distributed memory system is to let each processor own $n / p$ vertices and all the outgoing edges from those vertices. We refer to this partitioning of the graph as ' $1 \mathrm{D}$ partitioning', as it translates to the one-dimensional decomposition of the adjacency matrix corresponding to the graph.

The general schematic of the level-synchronous parallel BFS algorithm can be modified to work in a distributed scenario with 1D partitioning as well. Algorithm 2 gives the pseudo-code for BFS on a cluster of multicore or multithreaded processors. The distance array is distributed among processes. Every process only maintains the status of vertices it owns, and so the traversal loop becomes an edge aggregation phase. We can utilize multithreading within a process to enumerate the adjacencies. However, only the owner process of a vertex can identify whether it is newly visited or not. Thus, all the adjacencies of the vertices in the current frontier need to be sent to their corresponding owner process, which happens in the All-to-all communication step (line 21) of the algorithm. Note that the only thread-level synchronization required is due to the barriers. The rest of the steps such as buffer packing and unpacking can be performed by the threads in a data-parallel manner. The key aspects to note in this algorithm, in comparison to the serial level-synchronous algorithm (Algorithm 1), is the extraneous computation (and communication) introduced due to the distributed graph scenario: creating the message buffers of cumulative size $O(m)$ and the All-to-all communication step.

\subsection{BFS with 2D Partitioning}

We next describe a parallel BFS approach that directly works with the sparse adjacency matrix of the graph. Factoring out the underlying algebra, each BFS iteration is computationally equivalent to a sparse matrix-sparse vector multiplication (SpMSV). Let $A$ denote the adjacency matrix of the graph, represented in a sparse boolean format, $x_{k}$ denotes the $k$ th frontier, represented as a sparse vector with integer variables. It is easy to see that the exploration of level $k$ in BFS is algebraically equivalent to $x_{k+1} \leftarrow A^{\top} \otimes x_{k} \odot \overline{\bigcup_{i=1}^{x_{i}}}$ (we will omit the transpose and assume that the input is pre-transposed for the rest of this section). The syntax $\otimes$ denotes the matrix-vector multiplication operation on a special,$\odot$ denotes element-wise multiplication, and overline represents the complement operation. In other words, $\bar{v}_{i}=0$ for $v_{i} \neq 0$ and $\bar{v}_{i}=1$ for $v_{i}=0$. This algorithm becomes deterministic with the use of (select,max)-semiring, because the parent is always chosen to be the vertex with the highest label. The algorithm does not have to store the previous frontiers explicitly as multiple sparse vectors. In practice, it keeps a dense parents $=\bigcup_{i=1}^{x_{i}}$ array, which is more space efficient and easier to update.

Our sparse matrix approach uses the alternative $2 \mathrm{D}$ decomposition of the adjacency matrix of the graph. Consider the simple checkerboard partitioning, where processors are logically organized on a square $p=p_{r} \times p_{c}$ mesh, indexed by their row and column indices so that the $(i, j)$ th processor is denoted by $P(i, j)$. Edges and vertices (sub-matrices) are assigned to processors according to a $2 \mathrm{D}$ block decomposition. 


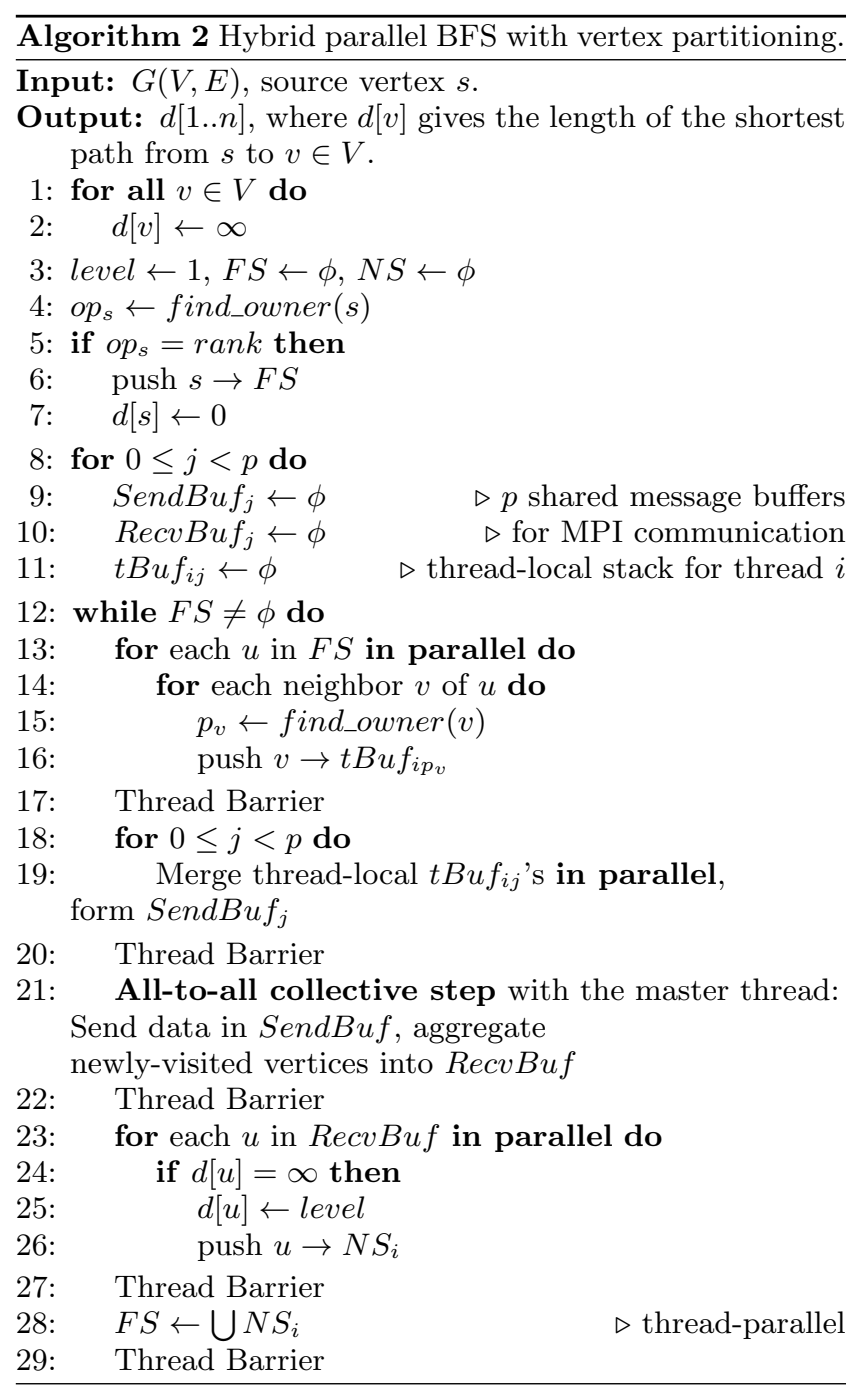

Each node stores a sub-matrix of dimensions $\left(n / p_{r}\right) \times\left(n / p_{c}\right)$ in its local memory.

Algorithm 3 gives the high-level pseudocode of our parallel algorithm for BFS on 2D-distributed graphs. This algorithm implicitly computes the "breadth-first spanning tree" by returning a dense parents array. The inner loop block starting in line 4 performs a single level traversal. All vectors and the input matrix are 2D-distributed as illustrated in Figure 1. $f$, which is initially an empty sparse vector, represents the current frontier. $t$ is an sparse vector that holds the temporary parent information for that iteration only. For a distributed vector $v$, the syntax $v_{i j}$ denotes the local $n / p$ sized piece of the vector owned by the $P(i, j)$ th processor. The syntax $v_{i}$ denotes the hypothetical $n / p_{r}$ or $n / p_{c}$ sized piece of the vector collectively owned by all the processors along the $i$ th processor row $P(i,:)$ or column $P(:, i)$.

Each computational step can be efficiently parallelized with multithreading. The multithreading of the SpMSV operation in line 7 naturally follows the splitting of the local sparse matrix data structure row-wise to $t$ pieces. The vector operations in lines $9-10$ is parallelized simply by exploiting loop-level parallelism.

TRAnsposeVECTOR redistributes the vector so that the subvector owned by the $i$ th processor row is now owned by the $i$ th processor column. In the case of $p_{r}=p_{c}=\sqrt{p}$, the
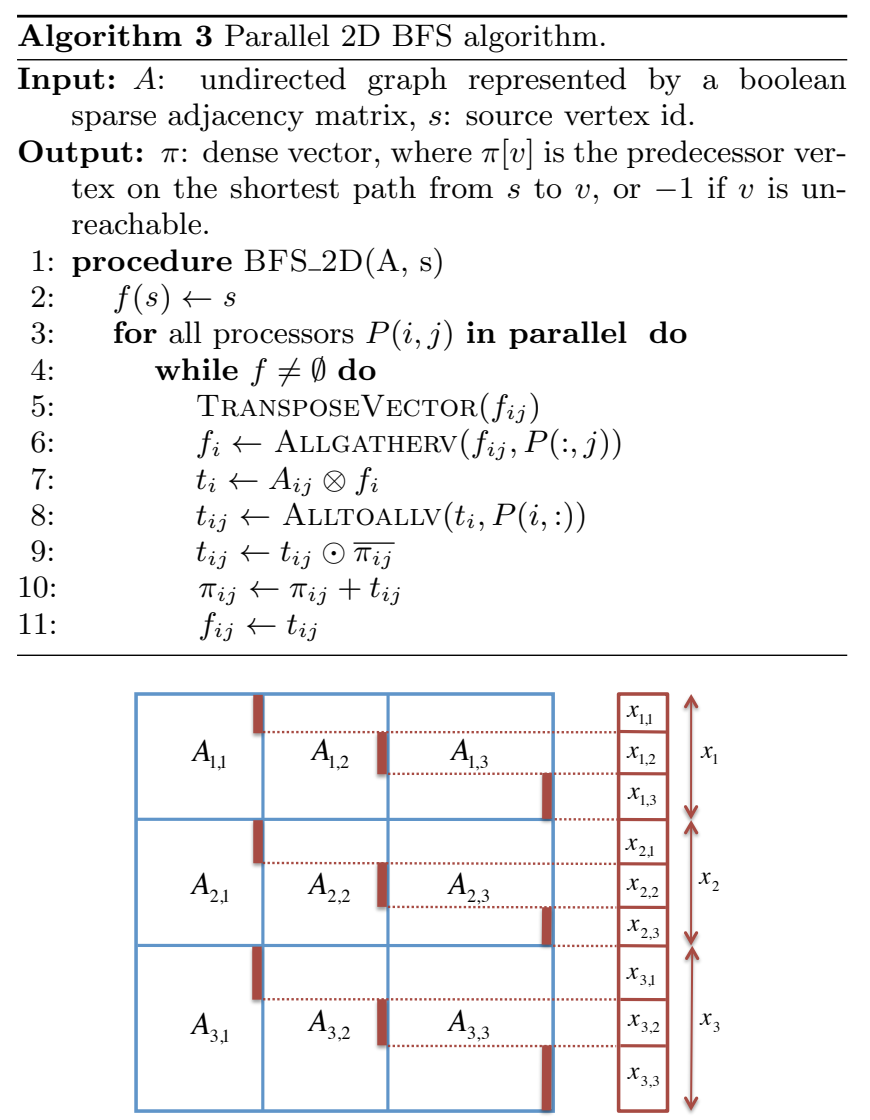

Figure 1: 2D vector distribution illustrated by interleaving it with the matrix distribution.

operation is simply a pairwise exchange between $P(i, j)$ and $P(j, i)$. In the more general case, it involves an all-to-all exchange among processor groups of size $p_{r}+p_{c}$.

$\operatorname{Allgather}\left(f_{i j}, P(:, j)\right)$ syntax denotes that subvectors $f_{i j}$ for $j=1, \ldots, p_{r}$ is accumulated at all the processors on the $j$ th processor column. Similarly, $\operatorname{Alltodulv}\left(t_{i}, P(i,:)\right)$ denotes that each processor scatters the corresponding piece of its intermediate vector $t_{i}$ to its owner, along the $i$ th processor row.

There are two distinct communication phases in a BFS algorithm with 2D partitioning: a pre-computation "expand" phase over the processor column ( $p_{r}$ processes), and a postcomputation "fold" phase over the processor row $\left(p_{c}\right.$ processes).

In 1D decomposition, the partitioning of frontier vectors naturally follows the vertices. In $2 \mathrm{D}$ decomposition, however, vertex ownerships are more flexible. One practice is to distribute the vector entries only over one processor dimension $\left(p_{r}\right.$ or $\left.p_{c}\right)$ [23], for instance the diagonal processors if using a square grid $\left(p_{r}=p_{c}\right)$, or the first processors of each processor row. This approach is mostly adequate for sparse matrix-dense vector multiplication (SpMV), since no local computation is necessary for the "fold" phase after the reduce step, to which all the processors contribute. For SpMSV, however, distributing the vector to only a subset of processors causes severe imbalance as we show in Section 4.3.

A more scalable and storage-efficient approach is to let each processor have approximately the same number of vertices. In this scheme, which we call the " $2 \mathrm{D}$ vector dis- 
tribution" in contrast to the " $1 \mathrm{D}$ vector distribution", we still respect the two-dimensional processor grid. In other words, the $2 \mathrm{D}$ vector distribution matches the matrix distribution. Each processor row (except the last) is responsible for $t=\left\lfloor n / p_{r}\right\rfloor$ elements. The last processor row gets the remaining $n-\left\lfloor n / p_{r}\right\rfloor\left(p_{r}-1\right)$ elements Within the processor row, each processor (except the last) is responsible for $l=\left\lfloor t / p_{c}\right\rfloor$ elements. In that sense, all the processors on the $i$ th processor row contribute to the storage of the vertices that are numbered from $v_{i p_{r}+1}$ to $v_{(i+1) p_{r}}$. The only downside of the this approach is that the "expand" phase becomes an all-gather operation (within the processor column) instead of the cheaper broadcast.

\section{IMPLEMENTATION DETAILS}

\subsection{Graph Representation}

For the graph-based BFS implementation, we use a 'compressed sparse row' (CSR)-like representation for storing adjacencies. All adjacencies of a vertex are sorted and compactly stored in a contiguous chunk of memory, with adjacencies of vertex $i+1$ next to the adjacencies of $i$. For directed graphs, we store only edges going out of vertices. Each edge (rather the adjacency) is stored twice in case of undirected graphs. An array of size $n+1$ stores the start of each contiguous vertex adjacency block. We use 64-bit integers to represent vertex identifiers. This representation is space-efficient in the sense that the aggregate storage for the distributed data structure is on the same order as the storage that would be needed to store the same data structure serially on a machine with large enough memory. Since our graph is static, linked data structures such as adjacency lists would incur more cache misses without providing any additional benefits.

On the contrary, a CSR-like representation is too wasteful for storing sub-matrices after $2 \mathrm{D}$ partitioning. The aggregate memory required to locally store each submatrix in CSR format is $O(n \sqrt{p}+m)$, while storing the whole matrix in CSR format would only take $O(n+m)$. Consequently, a strictly $O(m)$ data structure (possibly with fast indexing support) is required. One such data structure, doublycompressed sparse columns (DCSC), has been introduced before [7] for hypersparse matrices that arise after $2 \mathrm{D}$ decomposition. DCSC for BFS consists of an array IR of row ids (size $m$ ), which is indexed by two parallel arrays of column pointers (CP) and column ids (JC). The size of these parallel arrays are on the order of the number of columns that has at least one nonzero $(n z c)$ in them.

For the hybrid 2D algorithm, we split the node local matrix rowwise to $t$ (number of threads) pieces. Each thread local $n /\left(p_{r} t\right) \times n / p_{c}$ sparse matrix is stored in DCSC format.

A compact representation of the frontier vector is also important. It should be represented in a sparse format, where only the indices of the non-zeros are stored. We use a stack in the $1 \mathrm{D}$ implementation and a sorted sparse vector in the $2 \mathrm{D}$ implementation. Any extra data that are piggybacked to the frontier vectors adversely affect the performance, since the communication volume of the BFS benchmark is directly proportional to the size of this vector.

\subsection{Shared memory computation}

There are two potential bottlenecks to multithreaded parallel scaling in Algorithm 2 on our target architectural plat- forms (multicore systems with modest levels of thread-level parallelism). Consider pushes of newly-visited vertices to the stack NS. A shared stack for all threads would involve thread contention for every insertion. We use thread-local stacks (indicated by $N S_{i}$ in the algorithm) for storing these vertices, and merging them at the end of each iteration to form $F S$, the frontier stack. Note that the total number of queue pushes is bounded by $n$, the number of vertices in the graph. Hence, the cumulative memory requirement for these stacks is bounded, and the additional computation performed due to merging would be $O(n)$. Our choice is different from the approaches taken in prior work (such as specialized set data structures [25] or a shared queue with atomic increments [1]). For multithreaded experiments conducted in this study, we found that our choice does not limit performance, and the copying step constitutes a minor overhead, less than $3 \%$ of the execution time.

Next, consider the distance checks (lines 24-25) and updates in Algorithm 2. This is typically made atomic to ensure that a new vertex is added only once to the stack $N S$. We note that the BFS algorithm is still correct (but the output is non-deterministic) even if a vertex is added multiple times, as the distance value is guaranteed to be written correctly after the thread barrier and memory fence at the end of a level of exploration. Cache coherence further ensures that the correct value may propagate to other cores once it is updated. We observe that we actually perform a very small percentage of additional insertions (less than $0.5 \%$ ) for all the graphs we experimented with at six-way threading. This lets us avert the issue of non-scaling atomics across multi-socket configurations [1]. This optimization was also considered by Leiserson et al. [25] (termed "benign races") for insertions to their bag data structure.

For the $2 \mathrm{D}$ algorithm, the computation time is dominated by the sequential SpMSV operation in line 7 of Algorithm 3. This corresponds to selection, scaling and finally merging columns of the local adjacency matrix that are indexed by the nonzeros in the sparse vector. Computationally, we form the union $\bigcup A_{i j}(:, k)$ for all $k$ where $f_{i}(k)$ exists.

We explored multiple methods of forming this union. The first option is to use a priority-queue of size $n n z\left(f_{i}\right)$ and perform a unbalanced multiway merging. While this option has the advantage of being memory-efficient and automatically creating a sorted output, the extra logarithmic factor hurts the performance at small concurrencies, even after using a highly optimized cache-efficient heap. The cumulative requirement for these heaps are $O(m)$. The second option is to use a sparse accumulator (SPA) [18] which is composed of a dense vector of values, a bit mask representing the "occupied" flags, and a list that keeps the indices of existing elements in the output vector. The SPA approach proved to be faster for lower concurrencies, although it has disadvantages such as increasing the memory footprint due to the temporary dense vectors, and having to explicitly sort the indices at the end of the iteration. Our microbenchmarks [9] revealed a transition point around 10000 cores (for flat MPI version) after which the priority-queue method is more efficient, both in terms of speed and memory footprint. Our final algorithm is therefore a polyalgorithm depending on the concurrency.

\subsection{Distributed-memory parallelism}

We use the MPI message-passing library to express the 
inter-node communication steps. In particular, we extensively utilize the collective communication routines Alltoallv, Allreduce, and Allgatherv.

Our 2D implementation relies on the linear-algebraic primitives of the Combinatorial BLAS framework [8], with certain BFS-specific optimizations enabled.

We chose to distribute the vectors over all processors instead of only a subset (the diagonal processors in this case) of processors. In SpMSV, the accumulation of sparse contributions requires the diagonal processor to go through an additional local merging phase, during which all other processors on the processor row sit idle, causing severe load imbalance [9]. Distributing the vectors over all processors (2D vector distribution) remedies this problem and we observe almost no load imbalance in that case.

\subsection{Load-balancing traversal}

We achieve a reasonable load-balanced graph traversal by randomly shuffling all the vertex identifiers prior to partitioning. This leads to each process getting roughly the same number of vertices and edges, regardless of the degree distribution. An identical strategy is also employed in the Graph 500 BFS benchmark. The downside to this is that the edge cut can be potentially as high as an average random balanced cut, which can be $O(m)$ for several random graph families [34].

\section{ALGORITHM ANALYSIS}

The RAM and PRAM models capture asymptotic work and parallel execution time complexity with extremely simple underlying assumptions of the machine model, and are inadequate to analyze and contrast parallel BFS algorithmic variants on current parallel systems. For instance, the PRAM asymptotic time complexity for a level-synchronous parallel BFS is $O(D)$ (where $D$ is the diameter of the graph), and the work complexity is $O(m+n)$. These terms do not provide any realistic estimate of performance on current parallel systems.

We propose a simple linear model to capture the cost of regular (unit stride or fixed-stride) and irregular memory references to various levels of the memory hierarchy, as well as to succinctly express inter-processor MPI communication costs. We use the terms $\alpha$ and $\beta$ to account for the latency of memory accesses and the transfer time per memory word (i.e., inverse of bandwidth) respectively. Further, we use $\alpha_{L}$ to indicate memory references to local memory, and $\alpha_{N}$ to denote message latency over the network (remote memory accesses). The bandwidth terms can also be similarly defined. To account for the differing access times to various levels of the memory hierarchy, we additionally qualify the $\alpha$ term to indicate the size of the data structure (in memory words) that is being accessed. $\alpha_{L, x}$, for instance, would indicate the latency of memory access to a memory word in a logically-contiguous memory chunk of size $x$ words. Similarly, to differentiate between various inter-node collective patterns and algorithms, we qualify the network bandwidth terms with the communication pattern. For instance, $\beta_{N, p 2 p}$ would indicate the sustained memory bandwidth for point-to-point communication, $\beta_{N, a 2 a}$ would indicate the sustained memory bandwidth per node in case of an all-to-all communication scenario, and $\beta_{N, a g}$ would indicate the sustained memory bandwidth per node for an allgather operation.
Using synthetic benchmarks, the values of $\alpha$ and $\beta$ defined above can be calculated offline for a particular parallel system and software configuration. The programming model employed, the messaging implementation used, the compiler optimizations employed are some software factors that determine the various $\alpha$ and $\beta$ values.

\subsection{Analysis of the 1D Algorithm}

Consider the locality characteristics of memory references in the level-synchronous BFS algorithm. Memory traffic comprises touching every edge once (i.e., accesses to the adjacency arrays, cumulatively $m$ ), reads and writes from/to the frontier stacks $(n)$, distance array checks ( $m$ irregular accesses to an array of size $n$ ) and writes ( $n$ accesses to $d$ ). The complexity of the 1D BFS algorithm in our model is thus $m \beta_{L}$ (cumulative adjacency accesses) $+n \alpha_{n}$ (accesses to adjacency array pointers) $+m \alpha_{n}$ (distance checks/writes).

In the parallel case with $1 \mathrm{D}$ vertex and edge partitioning, the number of local vertices $n_{l o c}$ is approximately $n / p$ and the number of local edges is $m / p$. The local memory reference cost is given by $\frac{m}{p} \beta_{L}+\frac{n}{p} \alpha_{L, n / p}+\frac{m}{p} \alpha_{L, n / p}$. The distance array checks thus constitute the substantial fraction of the execution time, since the $\alpha_{L, n / p}$ term is significantly higher than the $\beta_{L}$ term. One benefit of the distributed approach is the array size for random accesses reduces from $n$ to $n / p$, and so the cache working set of the algorithm is substantially lower. Multithreading within a node (say, $t$-way threading) has the effect of reducing the number of processes and increasing the increasing the process-local vertex count by a factor of $t$.

The remote memory access costs are given by the All-to-all step, which involves a cumulative data volume of $m(p-1) / p$ words sent on the network. For a random graph with a uniform degree distribution, each process would send every other process roughly $m / p^{2}$ words. This value is typically large enough that the bandwidth component dominates over the latency term. Since we randomly shuffle the vertex identifiers prior to execution of BFS, these communication bounds hold true in case of the synthetic random networks we experimented with in this paper. Thus, the per-node communication cost is given by $p \alpha_{N}+\frac{m}{p} \beta_{N, a 2 a}(p)$. $\beta_{N, a 2 a}(p)$ is a function of the processor count, and several factors, including the interconnection topology, node injection bandwidth, the routing protocol, network contention, etc. determine the sustained per-node bandwidth. For instance, if nodes are connected in a 3D torus, it can be shown that bisection bandwidth scales as $p^{2 / 3}$. Assuming all-toall communication scales similarly, the communication cost can be revised to $p \alpha_{N}+\frac{m}{p} p^{1 / 3} \beta_{N, a 2 a}$. If processors are connected via a ring, then $p \alpha_{N}+\frac{m}{p} p \beta_{N, a 2 a}$ would be an estimate for the all-to-all communication cost, essentially meaning no parallel speedup.

\subsection{Analysis of the 2D Algorithm}

Consider the general 2D processor grid of $p_{r} \times p_{c}$. The size of the local adjacency matrix is $n / p_{r} \times n / p_{c}$. The number of memory references is the same as the $1 \mathrm{D}$ case, cumulatively over all processors. However, the cache working set is bigger, because the sizes of the local input (frontier) and output vectors are $n / p_{r}$ and $n / p_{c}$, respectively. The local memory reference cost is given by $\frac{m}{p} \beta_{L}+\frac{n}{p} \alpha_{L, n / p_{c}}+\frac{m}{p} \alpha_{L, n / p_{r}}$. The higher number of cache misses associated with larger working sets is perhaps the primary reason for the relatively 
higher computation costs of the $2 \mathrm{D}$ algorithm.

Most of the costs due to remote memory accesses is concentrated in two operations. The expand phase is characterized by an Allgatherv operation over the processor column (of size $p_{r}$ ) and the fold phase is characterized by an Alltoallv operation over the processor row (of size $p_{c}$ ).

The aggregate input to the Allgatherv step is $O(n)$ over all iterations. However, each processor receives a $1 / p_{c}$ portion of it, meaning that frontier subvector gets replicated along the processor column. Hence, the per node communication cost is $p_{r} \alpha_{N}+\frac{n}{p_{c}} \beta_{N, a g}\left(p_{r}\right)$. This replication can be partially avoided by performing an extra round of communication where each processor individually examines its columns and broadcasts the indices of its nonempty columns. However, this extra step does not decrease the asymptotic complexity for graphs that do not have good separators.

The aggregate input to the Alltoallv step can be as high as $O(m)$, although the number is lower in practice due to in-node aggregation of newly discovered vertices that takes place before the communication. Since each processor receives only a $1 / p$ portion of this data, the remote costs due to this step are at most $p_{c} \alpha_{N}+\frac{m}{p} \beta_{N, a 2 a}\left(p_{c}\right)$.

We see that for large $p$, the expand phase is likely to be more expensive than the fold phase. Our analysis successfully captures that the relatively lower communication costs of the $2 \mathrm{D}$ algorithm by representing $\beta_{N, x}$ as a function of the processor count.

\section{EXPERIMENTAL STUDIES}

We have extensively evaluated the performance of our two parallel BFS schemes. We experiment with two parallel programming models: "Flat MPI" with one process per core, and a hybrid implementation with one or more MPI processes within a node. We use synthetic graphs based on the R-MAT random graph model [10], as well as a largescale real world graph that represents a web crawl of the UK domain [6] (uk-union) that has 133 million vertices and 5.5 billion edges. The R-MAT generator creates networks with skewed degree distributions and a very low graph diameter. We set the R-MAT parameters $a, b, c$, and $d$ to $0.59,0.19,0.19,0.05$ respectively. These parameters are identical to the ones used for generating synthetic instances in the Graph 500 BFS benchmark. R-MAT graphs make for interesting test instances: traversal load-balancing is nontrivial due to the skewed degree distribution, the graphs lack good separators, and common vertex relabeling strategies are also expected to have a minimal effect on cache performance. The diameter of the uk-union graph is significantly higher $(\approx 140)$ than R-MAT's (less than 10 ), allowing us to access the sensitivity of our algorithms with respect to the number of synchronizations. We use undirected graphs for all our experiments, but the BFS approaches can work with directed graphs as well.

To compare performance across multiple systems using a rate analogous to the commonly-used floating point operations/second, we normalize the serial and parallel execution times by the number of edges visited in a BFS traversal and present a 'Traversed Edges Per Second' (TEPS) rate. For a graph with a single connected component (or one strongly connected component in case of directed networks), the baseline BFS algorithm would visit every edge twice (once in case of directed graphs). We only consider traversal execution times from vertices that appear in the large
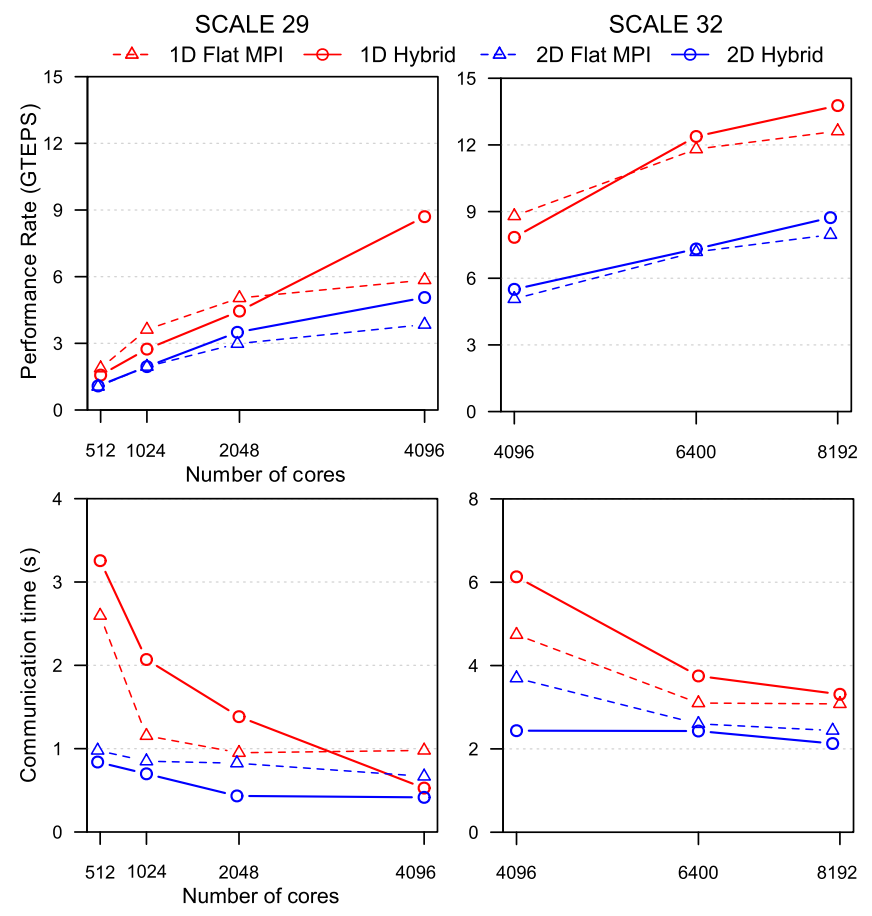

Figure 2: BFS 'strong scaling' results on Franklin for R-MAT graphs: Performance rate achieved (in GTEPS) on increasing the number of processors (top), and the corresponding average inter-node MPI communication time (in seconds, bottom).

component, compute the mean search time (harmonic mean of TEPS) using at least 16 randomly-chosen sources vertices for each benchmark graph, and normalize the time by the cumulative number of edges visited to get the TEPS rate. As suggested by the Graph 500 benchmark, we first symmetrize the input to model undirected graphs. For TEPS calculation, we only count the number of edges in the original directed graph, despite visiting symmetric edges as well. The performance rate captures both the communication time and the computation time. For R-MAT graphs, the default edge count to vertex ratio is set to 16 (which again corresponds to the Graph 500 default setting), but we also vary the ratio of edge to vertex counts in some of our experiments.

We collect performance results on two large systems: 'Hopper', a 6392-node Cray XE6 and 'Franklin', a 9660-node Cray XT4. Both supercomputers are located at NERSC, Lawrence Berkeley National Laboratory. Each XT4 node contains a quad-core $2.3 \mathrm{GHz}$ AMD Opteron processor, which is tightly integrated to the XT4 interconnect via a Cray SeaStar2 ASIC through a HyperTransport (HT) 2 interface capable of $6.4 \mathrm{~GB} / \mathrm{s}$. The SeaStar routing chips are interconnected in a 3D torus topology, and each link is capable of $7.6 \mathrm{~GB} / \mathrm{s}$ peak bidirectional bandwidth. Each XE6 node contains two twelve-core $2.1 \mathrm{GHz}$ AMD Opteron processors, integrated to the Cray Gemini interconnect through HT 3 interfaces. Each Gemini chip is capable of $9.8 \mathrm{~GB} / \mathrm{s}$ bandwidth. Two XE6 nodes share a Gemini chip as opposed to the one-to-one relationship on XT4. The effective bisection bandwidth of XE6 is slightly lower (ranging from 1-20\%, depending on the bisection dimension) than XT4's. Each twelve-core 'MagnyCours' die is essentially composed of two 6-core NUMA nodes. More information on the architectures 

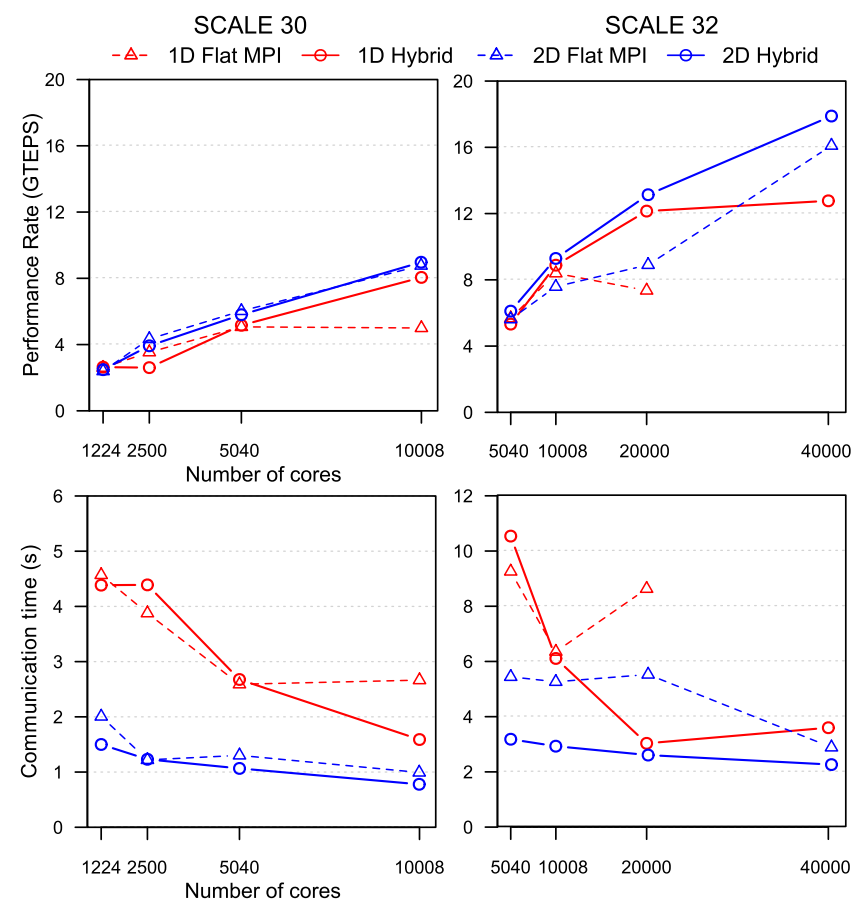

Figure 3: BFS 'strong scaling' results on Hopper for R-MAT graphs: Performance rate achieved (in GTEPS) on increasing the number of processors (top), and the corresponding average inter-node MPI communication time (in seconds, bottom).

can be found in the extended version [9].

We used the GNU C/C++ compilers (v4.5) for compiling both implementations. The $1 \mathrm{D}$ versions are implemented in $\mathrm{C}$, whereas the $2 \mathrm{D}$ codes are implemented in $\mathrm{C}++$. We use Cray's MPI implementation, which is based on MPICH2. For intra-node threading, we use the GNU OpenMP library.

Figure 2 shows 'strong scaling' of our algorithms' performance (higher is better) on Franklin. We see that the flat $1 \mathrm{D}$ algorithms are about $1.5-1.8 \times$ faster than the $2 \mathrm{D}$ algorithms on this architecture. The 1D hybrid algorithm, albeit slower than the flat 1D algorithm for smaller concurrencies, starts to perform significantly faster for larger concurrencies. We attribute this effect partially to bisection bandwidth saturation, and partially to the saturation of the network interface card when using more cores (leading to more outstanding communication requests) per node. The $2 \mathrm{D}$ hybrid algorithm tends to outperform the flat 2D algorithm, but can not compete with the $1 \mathrm{D}$ algorithms on this architecture as it spends significantly more time in computation. This is due to relatively larger cache working sizes, as captured by our model in Section 5 .

The communication costs, however, tell a different story about the relative competitiveness of our algorithms. 2D algorithms consistently spend less time (30-60\% for scale 32 ) in communication, compared to their relative $1 \mathrm{D}$ algorithms. This is also expected by our analysis, as smaller number of participating processors in collective operations tend to result in faster communication times, with the same amount of data. The hybrid $1 \mathrm{D}$ algorithm catches up with the flat $2 \mathrm{D}$ algorithm at large concurrencies for the smaller (scale 29) dataset, but still lags behind the hybrid 2D algorithm.

Strong scaling results on Hopper are shown in Figure 3.
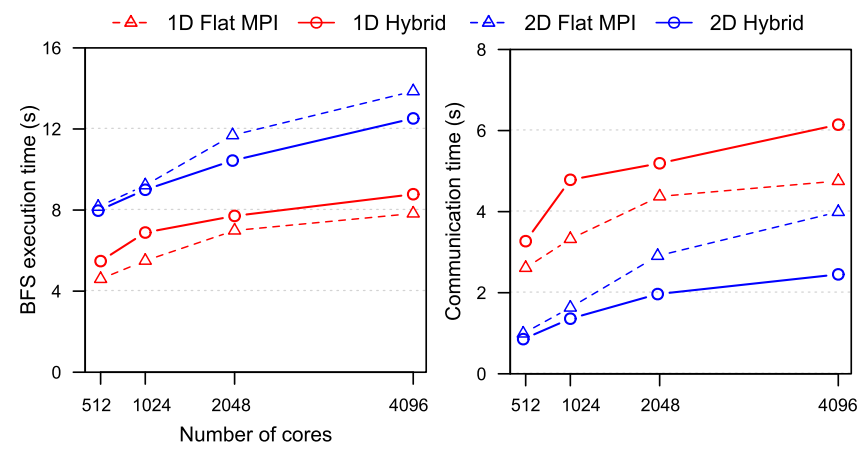

Figure 4: BFS 'weak scaling' results on Franklin for Graph 500 R-MAT graphs: Mean search time (left) and MPI communication time (right) on fixed problem size per core (each core has $\approx 17 \mathrm{M}$ edges). In both plots, lower is better.

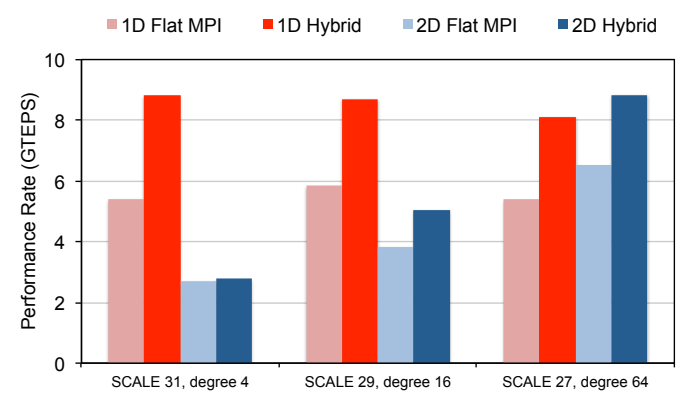

Figure 5: BFS GTEPS performance rate achieved on 4096 cores of Franklin by varying the average vertex degree for R-MAT graphs.

In contrast to Franklin results, the 2D algorithms perform better than their 1D counterparts. The more sophisticated Magny-Cours chips of Hopper are clearly faster in integer calculations, while the overall bisection bandwidth has not kept pace. We did not execute the flat $1 \mathrm{D}$ algorithm on $40 \mathrm{~K}$ cores as the communication times already started to increase when going from $10 \mathrm{~K}$ to $20 \mathrm{~K}$ cores, consuming more than $90 \%$ of the overall execution time. In contrast, the percentage of time spent in communication for the $2 \mathrm{D}$ hybrid algorithm was less than $50 \%$ on $20 \mathrm{~K}$ cores.

"Weak scaling" performance results on Franklin are shown in Figure 4, where we fix the edges per processor to a constant value. To be consistent with prior literature, we present weak scaling results in terms of the time it takes to complete the BFS iterations, with ideal curve being a flat line. Interestingly, in this regime, the flat 1D algorithm performs better than the hybrid 1D algorithm, both in terms of overall performance and communication costs. The $2 \mathrm{D}$ algorithms, although performing much less communication than their 1D counterparts, come later in terms of overall performance on this architecture, due to their higher computation overheads.

Figure 5 shows the sensitivity of our algorithms to varying graph densities. In this experiment, we kept the number of edges per processor constant by varying the number of vertices as the average degree varies. The trend is obvious in that the performance margin between the $1 \mathrm{D}$ algorithms and the $2 \mathrm{D}$ algorithms increases in favor of the $1 \mathrm{D}$ algorithm as the graph gets sparser. The empirical data supports our 


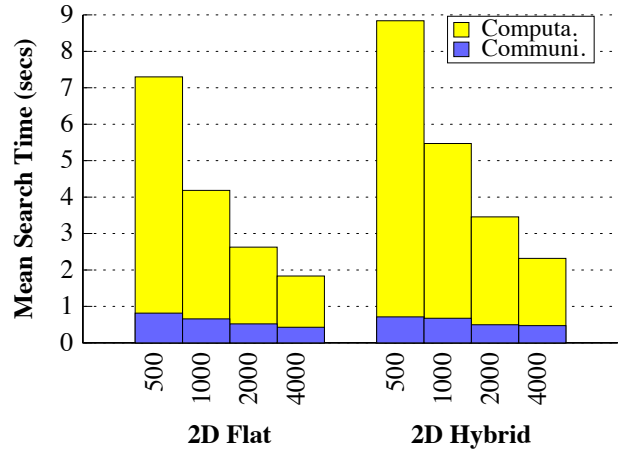

Figure 6: Running times of the 2D algorithms on the uk-union data set on Hopper (lower is better). The numbers on the $\mathrm{x}$-axis is the number of cores. The running times translate into a maximum of 3 GTEPS performance, achieving a $4 \times$ speedup when going from 500 to 4000 cores

analysis in Section 5, which stated that the $2 \mathrm{D}$ algorithm performance was limited by the local memory accesses to its relatively larger vectors. For a fixed number of edges, the matrix dimensions (hence the length of intermediate vectors) shrink as the graph gets denser, partially nullifying the cost of local cache misses.

We show the performance of our 2D algorithms on the real uk-union data set in Figure 6. We see that communication takes a very small fraction of the overall execution time, even on $4 \mathrm{~K}$ cores. This is a notable result because the uk-union dataset has a relatively high-diameter and the BFS takes approximately 140 iterations to complete. Since communication is not the most important factor, the hybrid algorithm is slower than flat MPI, as it has more intra-node parallelization overheads.

To compare our approaches with prior and reference distributed memory implementations, we experimented with the Parallel Boost Graph Library's (PBGL) BFS implementation [21] (Version 1.45 of the Boost library) and the reference MPI implementation (Version 2.1) of the Graph 500 benchmark [20]. On Franklin, our Flat 1D code is $2.72 \times$, $3.43 \times$, and $4.13 \times$ faster than the non-replicated reference MPI code on 512, 1024, and 2048 cores, respectively.

Since PBGL failed to compile on the Cray machines, we ran comparison tests on Carver, an IBM iDataPlex system with 400 compute nodes, each node having two quad-core Intel Nehalem processors. PBGL suffers from severe memory bottlenecks in graph construction that hinder scalable creation of large graphs. Our results are summarized in Table 1 for graphs that ran to completion. We are up to $16 \times$ faster than PBGL even on these small problem instances.

Extensive experimentation reveals that our single-node multithreaded BFS version (i.e., without the inter-node communication steps in Algorithm 2) is also extremely fast. We compare the Nehalem-EP performance results reported in the work by Agarwal et al. [1] with the performance on a single node of the Carver system (also Nehalem-EP), and notice that for R-MAT graphs with average degree 16 and 32 million vertices, our approach is nearly $1.30 \times$ faster. Our approach is also faster than BFS results reported by Leiserson et al. [25] on the KKt_power, Freescale1, and Cage14 test instances, by up to $1.47 \times$.
Table 1: Performance comparison with PBGL on Carver. The reported numbers are in MTEPS for R-MAT graphs with the same parameters as before. In all cases, the graphs are undirected and edges are permuted for load balance.

\begin{tabular}{c|c|c|c}
\multirow{2}{*}{ Core count } & \multirow{2}{*}{ Code } & \multicolumn{2}{|c}{ Problem Size } \\
\cline { 3 - 4 } & & Scale 22 & Scale 24 \\
\hline \multirow{2}{*}{128} & PBGL & 25.9 & 39.4 \\
& Flat 2D & 266.5 & 567.4 \\
\hline \multirow{2}{*}{256} & PBGL & 22.4 & 37.5 \\
& Flat 2D & 349.8 & 603.6
\end{tabular}

\section{CONCLUSIONS AND FUTURE WORK}

In this paper, we present a design-space exploration of distributed-memory parallel BFS, discussing two fast "hybridparallel" approaches for large-scale graphs. Our experimental study encompasses performance analysis on several largescale synthetic random graphs that are also used in the recently announced Graph 500 benchmark. The absolute performance numbers we achieve on the large-scale parallel systems Hopper and Franklin at NERSC are significantly higher than prior work. The performance results, coupled with our analysis of communication and memory access costs of the two algorithms, challenges conventional wisdom that fine-grained communication is inherent in parallel graph algorithms and necessary for achieving high performance [26].

We list below optimizations that we intend to explore in future work, and some open questions related to design of distributed-memory graph algorithms.

Exploiting symmetry in undirected graphs. If the graph is undirected, then one can save $50 \%$ space by storing only the upper (or lower) triangle of the sparse adjacency matrix, effectively doubling the size of the maximum problem that can be solved in-memory on a particular system. The algorithmic modifications needed to save a comparable amount in communication costs for BFS iterations is not well-studied.

Exploring alternate programming models. Partitioned global address space (PGAS) languages can potentially simplify expression of graph algorithms, as inter-processor communication is implicit. In future work, we will investigate whether our two new BFS approaches are amenable to expression using PGAS languages, and whether they can deliver comparable performance.

Reducing inter-processor communication volume with graph partitioning. An alternative to randomization of vertex identifiers is to use hypergraph partitioning software to reduce communication. Although hypergraphs are capable of accurately modeling the communication costs of sparse matrix-dense vector multiplication, SpMSV case has not been studied, which is potentially harder as the sparsity pattern of the frontier vector changes over BFS iterations. Interprocessor collective communication optimization. We conclude that even after alleviating the communication costs, the performance of distributed-memory parallel BFS is heavily dependent on the inter-processor collective communication routines All-to-all and Allgather. Understanding the bottlenecks in these routines at high process concurrencies, and designing network topology-aware collective algorithms is an interesting avenue for future research. 


\section{Acknowledgments}

This work was supported by the Director, Office of Science, U.S. Department of Energy under Contract No. DE-AC0205CH11231. Discussions with John R. Gilbert, Steve Reinhardt, and Adam Lugowski greatly improved our understanding of casting BFS iterations into sparse linear algebra. John Shalf and Nick Wright provided generous technical and moral support during the project.

\section{REFERENCES}

[1] V. Agarwal, F. Petrini, D. Pasetto, and D.A. Bader. Scalable graph exploration on multicore processors. In Proc. ACM/IEEE Conference on Supercomputing (SC10), November 2010.

[2] D. Ajwani, R. Dementiev, and U. Meyer. A computational study of external-memory BFS algorithms. In Proc. 17th annual ACM-SIAM Symposium on Discrete Algorithms (SODA '06), pages 601-610, January 2006.

[3] D. Ajwani and U. Meyer. Design and engineering of external memory traversal algorithms for general graphs. In J. Lerner, D. Wagner, and K.A. Zweig, editors, Algorithmics of Large and Complex Networks: Design, Analysis, and Simulation, pages 1-33. Springer, 2009.

[4] D.A. Bader and K. Madduri. Designing multithreaded algorithms for breadth-first search and st-connectivity on the Cray MTA-2. In Proc. 35th Int'l. Conf. on Parallel Processing (ICPP 2006), pages 523-530, August 2006.

[5] J. Barnat, L. Brim, and J. Chaloupka. Parallel breadth-first search LTL model-checking. In Proc. 18th IEEE Int'l. Conf. on Automated Software Engineering, pages 106-115, October 2003.

[6] P. Boldi and S. Vigna. The WebGraph framework I: Compression techniques. In Proc. 13th Int'l. World Wide Web Conference (WWW 2004), pages 595-601, 2004.

[7] A. Buluç and J.R. Gilbert. On the representation and multiplication of hypersparse matrices. In Proc. Int'l Parallel and Distributed Processing Symp. (IPDPS 2008), pages 1-11. IEEE Computer Society, 2008.

[8] A. Buluç and J.R. Gilbert. The Combinatorial BLAS: Design, implementation, and applications. The International Journal of High Performance Computing Applications, Online first, 2011.

[9] A. Buluç and K. Madduri. Parallel breadth-first search on distributed memory systems. Technical Report LBNL-4769E, Lawrence Berkeley National Laboratory, 2011.

[10] D. Chakrabarti, Y. Zhan, and C. Faloutsos. R-MAT: A recursive model for graph mining. In Proc. 4th SIAM Intl. Conf. on Data Mining (SDM), Orlando, FL, April 2004. SIAM.

[11] A. Chan, F. Dehne, and R. Taylor. CGMGRAPH/CGMLIB: Implementing and testing CGM graph algorithms on PC clusters and shared memory machines. Int'l. Journal of High Performance Comput. Appl., 19(1):81-97, 2005.

[12] G. Cong, G. Almasi, and V. Saraswat. Fast PGAS implementation of distributed graph algorithms. In
Proc. ACM/IEEE Conference on Supercomputing (SC10), November 2010.

[13] G. Cong and K. Makarychev. Improving memory access locality for large-scale graph analysis applications. In Proc. 22nd Intl. Parallel and Distributed Computing and Communication Systems (PDCCS 2009), pages 121-127, September 2009.

[14] T. H. Cormen, C. E. Leiserson, and R. L. Rivest. Introduction to Algorithms. MIT Press, Inc., Cambridge, MA, 1990.

[15] E. Cuthill and J. McKee. Reducing the bandwidth of sparse symmetric matrices. In Proc. 24th ACM Annual Conf./Annual Meeting, pages 157-172, 1969.

[16] N. Edmonds, J. Willcock, T. Hoefler, and A. Lumsdaine. Design of a large-scale hybrid-parallel graph library. In International Conference on High Performance Computing, Student Research Symposium, Goa, India, December 2010. To appear.

[17] H. Gazit and G.L. Miller. An improved parallel algorithm that computes the BFS numbering of a directed graph. Information Processing Letters, 28(2):61-65, 1988.

[18] J.R. Gilbert, C. Moler, and R. Schreiber. Sparse matrices in Matlab: Design and implementation. SIAM Journal of Matrix Analysis and Applications, 13(1):333-356, 1992.

[19] J.R. Gilbert, S. Reinhardt, and V.B. Shah. A unified framework for numerical and combinatorial computing. Computing in Science and Engineering, 10(2):20-25, 2008.

[20] The Graph 500 List. http://www.graph500.org, last accessed April 2011.

[21] D. Gregor and A. Lumsdaine. Lifting sequential graph algorithms for distributed-memory parallel computation. In Proc. 20th ACM SIGPLAN Conf. on Object Oriented Programming, Systems, Languages, and Applications (OOPSLA), pages 423-437, October 2005.

[22] P. Harish and P.J. Narayanan. Accelerating large graph algorithms on the GPU using CUDA. In Proc. 14 th Int'l. Conf. on High-Performance Computing (HiPC), pages 197-208, dec 2007.

[23] B. Hendrickson, R.W. Leland, and S. Plimpton. An efficient parallel algorithm for matrix-vector multiplication. International Journal of High Speed Computing, 7(1):73-88, 1995.

[24] R.E. Korf and P. Schultze. Large-scale parallel breadth-first search. In Proc. 20th National Conf. on Artificial Intelligence (AAAI'05), pages 1380-1385, July 2005.

[25] C.E. Leiserson and T.B. Schardl. A work-efficient parallel breadth-first search algorithm (or how to cope with the nondeterminism of reducers). In Proc. 22nd ACM Symp. on Parallism in Algorithms and Architectures (SPAA '10), pages 303-314, June 2010.

[26] A. Lumsdaine, D. Gregor, B. Hendrickson, and J.W. Berry. Challenges in parallel graph processing. Parallel Processing Letters, 17:5-20, 2007.

[27] L. Luo, M. Wong, and W m. Hwu. An effective GPU implementation of breadth-first search. In Proc. 47 th Design Automation Conference (DAC '10), pages 52-55, June 2010. 
[28] G. Malewicz, M.H. Austern, A.J.C. Bik, J.C. Dehnert, I. Horn, N. Leiser, and G. Czajkowski. Pregel: a system for large-scale graph processing. In Proc. Int'l. Conf. on Management of Data (SIGMOD '10), pages 135-146, June 2010.

[29] D. Mizell and K. Maschhoff. Early experiences with large-scale XMT systems. In Proc. Workshop on Multithreaded Architectures and Applications (MTAAP'09), May 2009.

[30] R. Pearce, M. Gokhale, and N.M. Amato. Multithreaded asynchronous graph traversal for in-memory and semi-external memory. In Proc. 2010 ACM/IEEE Int'l. Conf. for High Performance Computing, Networking, Storage and Analysis (SC'10), pages 1-11, 2010.

[31] M.J. Quinn and N. Deo. Parallel graph algorithms. ACM Comput. Surv., 16(3):319-348, 1984.

[32] A.E. Reghbati and D.G. Corneil. Parallel computations in graph theory. SIAM Journal of Computing, 2(2):230-237, 1978.

[33] D.P. Scarpazza, O. Villa, and F. Petrini. Efficient Breadth-First Search on the Cell/BE processor. IEEE Transactions on Parallel and Distributed Systems, 19(10):1381-1395, 2008.

[34] G.R. Schreiber and O.C. Martin. Cut size statistics of graph bisection heuristics. SIAM Journal on Optimization, 10(1):231-251, 1999.

[35] J. Ullman and M. Yannakakis. High-probability parallel transitive closure algorithms. In Proc. 2nd Annual Symp. on Parallel Algorithms and Architectures (SPAA 1990), pages 200-209, July 1990.

[36] S. Williams, L. Oliker, R. Vuduc, J. Shalf, K. Yelick, and J. Demmel. Optimization of sparse matrix-vector multiplication on emerging multicore platforms. Parallel Computing, 35(3):178-194, 2009.

[37] Y. Xia and V.K. Prasanna. Topologically adaptive parallel breadth-first search on multicore processors. In Proc. 21st Int'l. Conf. on Parallel and Distributed Computing Systems (PDCS'09), November 2009.

[38] A. Yoo, E. Chow, K. Henderson, W. McLendon, B. Hendrickson, and Ü. V. Çatalyürek. A scalable distributed parallel breadth-first search algorithm on BlueGene/L. In Proc. ACM/IEEE Conf. on High Performance Computing (SC2005), November 2005.

[39] K. You, J. Chong, Y. Yi, E. Gonina, C. Hughes, Y-K. Chen, W. Sung, and K. Kuetzer. Parallel scalability in speech recognition: Inference engine in large vocabulary continuous speech recognition. IEEE Signal Processing Magazine, 26(6):124-135, 2009. 